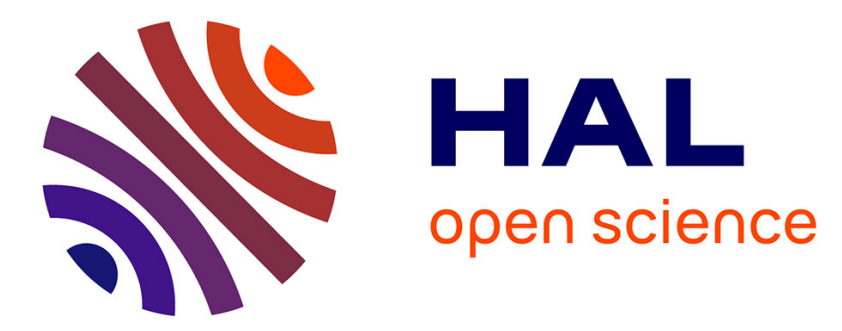

\title{
Overall elastoplastic behavior of anisotropic metal-matrix composites reinforced by aligned inclusions. Application to hydrided and irradiated Zircaloy-4 cladding tubes
}

Pierre-Guy Vincent, Stéphane Bourgeois, Yann Monerie

\section{To cite this version:}

Pierre-Guy Vincent, Stéphane Bourgeois, Yann Monerie. Overall elastoplastic behavior of anisotropic metal-matrix composites reinforced by aligned inclusions. Application to hydrided and irradiated Zircaloy-4 cladding tubes. Mechanics of Materials, 2010, 42 (2), pp.175-188. 10.1016/j.mechmat.2009.11.004 . hal-00461766

\section{HAL Id: hal-00461766 https://hal.science/hal-00461766}

Submitted on 16 Jun 2019

HAL is a multi-disciplinary open access archive for the deposit and dissemination of scientific research documents, whether they are published or not. The documents may come from teaching and research institutions in France or abroad, or from public or private research centers.
L'archive ouverte pluridisciplinaire HAL, est destinée au dépôt et à la diffusion de documents scientifiques de niveau recherche, publiés ou non, émanant des établissements d'enseignement et de recherche français ou étrangers, des laboratoires publics ou privés. 


\title{
Overall elastoplastic behavior of anisotropic metal-matrix composites reinforced by aligned inclusions. Application to hydrided and irradiated Zircaloy-4 cladding tubes
}

\author{
P.-G. Vincent ${ }^{\mathrm{a}}$, S. Bourgeois ${ }^{\mathrm{b}}$, Y. Monerie ${ }^{\mathrm{a}, \mathrm{c}, *}$ \\ ${ }^{a}$ Institut de Radioprotection et de Sûreté Nucléaire (IRSN), DPAM, BP3, 13115 Saint-Paul-Lez-Durance Cedex, France \\ ${ }^{\mathrm{b}}$ Laboratoire de Mécanique et d'Acoustique (CNRS-UPR7051), and Ecole Centrale Marseille, Technopôle de Château Gombert, 38 rue Joliot Curie, 13451 Marseille \\ ${ }^{c}$ Laboratoire de Micromécanique et d'Intégrité des Structures, IRSN-CNRS-UMII, B.P. 3, 13115 Saint-Paul-lez-Durance Cedex, France
}

\begin{abstract}
The present work aims at determining a micromechanical model for the elastoplastic behavior of anisotropic metal-matrix composites reinforced by aligned inclusions. The microstructure of the studied material - hydrided and irradiated Zircaloy-4 cladding tubes - is supposed to be made of an anisotropic and elastoplastic matrix (irradiated Zircaloy) containing aligned isotropic elastic inclusions (hydrides). The homogeneous behavior of this non-linear two-phase material is obtained by a linearization model based on the modified secant method (Ponte Castañeda and Willis, 1995). The behavior of the linear comparison composite is estimated by the model of Ponte Castañeda and Willis (1995). The anisotropy of the macroscopic behavior rises both from the shape of the inclusions and from the plastic anisotropy of the matrix. The model is compared to three-dimensional numerical simulations and experimental data.
\end{abstract}

\section{Introduction}

In the frame of its research program on nuclear fuel safety under accident conditions, the French "Institut de Radioprotection et de Sûreté Nucléaire" (IRSN) studies the constitutive materials of fuel rods in pressurized water reactors. Due to various physical phenomena appearing in these reactors (corrosion, irradiation, etc.), the materials constituting the fuel rods evolve continuously and their microstructure becomes progressively more and more complex. This evolution of microstructure is a strong specificity of the materials from the nuclear industry, which has motivated constant extensive research over the years.

\footnotetext{
* Corresponding author. Address: Institut de Radioprotection et de Sûreté Nucléaire (IRSN), DPAM, BP3, 13115 Saint-Paul-Lez-Durance Cedex, France. Tel.: +33 4421996 17; fax: +33 442199166 .

E-mail address: yann.monerie@irsn.fr (Y. Monerie).
}

The present work focuses on the material constitutive of cladding tubes containing nuclear fuel in pressurized water reactors: Zircaloy-4 (Zr). This material, as other zirconium base alloys, is widely used in light water reactors (fuel cladding tubes, fuel assembly spacer grids, guide tubes, etc.) because of its high resistance to corrosion, its small neutron absorption cross section and its good mechanical behavior at high temperature (Froes et al., 1996). The main limitation in the use of zirconium based alloys is the progressive appearance of hydride precipitates during their use inside the reactor, which drastically modifies their overall mechanical properties (Kearns, 1967; Northwood and Kosasih, 1983; Garde, 1989; Coleman and Hardie, 1966; Gómez et al., 2006). For Zircaloy cladding tubes, numerous experimental or theoretical works have been performed on: (i) the mechanisms of diffusion of hydrogen (Sawatzky, 1960; Marino, 1972; Varias and Massih, 2002), (ii) the mechanisms of dissolution and precipitation of hydrogen (Kearns, 1967; Kammenzind et al., 
1996; McMinn et al., 2000; Vizcaíno et al., 2002; Une and Ishimoto, 2003), and (iii) the hydride-induced embrittlement (Dutton et al., 1977; Coleman and Ambler, 1977; Huang and Mills, 1991; Arsène, 1997; Lufrano and Sofronis, 2000; Grange et al., 2000). However, very few works were devoted to the mechanical behavior of the Zircaloy before appearance of any damage, which is of primary interest during a reactivity initiated-type accident (see Fandeur (2001), Fandeur et al. (2001) and references therein for a phenomenological modelling). The present study is thus dedicated to the overall elastoplastic mechanical behavior of the hydrided and irradiated Zircaloy and its link with some meaningful micromechanical parameters.

Since Zircaloy is initialy elastoplastic and anisotropic (Delobelle et al., 1996), hydrided and irradiated Zircaloy has to be considered as an anisotropic non-linear twophase metal-matrix material. The overall constitutive behavior of this composite material is derived here from homogenization techniques. The parameters of the macroscopic model are the material properties, the morphology and the spatial distribution of each phase. Replacing the elastoplastic behavior of the matrix phase by a non-linear elastic behavior, one uses a generalization of the variational approach of Ponte Castañeda (1991) (or the so-called modified secant method, see Suquet (1995)) dedicated to composites with anisotropic phases, as presented in Ponte Castañeda and Suquet (1998). This approach, which improves the classical secant method (see for example Bornert and Suquet (2001)), introduces a linear comparison composite (LCC). The LCC has here the same geometry as the non-linear one, and its effective properties are estimated by using the result of Ponte Castañeda and Willis (1995). This estimate allows us to take into account both the shape and the distribution of the inclusions (hydride precipitates) inside the matrix.

The interest of the model is its ability to distinguish the anisotropy due to the initial matrix and as well as to the shape and the orientation of the inclusions. This work is an extension of the previous work of Li and Ponte Castañeda (1994) to the case of an anisotropic matrix and an isotropic distribution of the centers of the ellipsoidal inclusions.

This paper is organized as follows. In Section 2, the microstructure of the studied anisotropic metal-matrix composite is detailed. In Section 3, a new elastoplastic micromechanical-based model is derived. Section 4 deals both with the numerical implementation of the model and with the identification of its morphological parameter by numerical comparisons; several predictions offered by the model are shown. In Section 5, we review assorted applications of the hydrided and irradiated Zircaloy in an attempt to shed light on its practical assets.

\section{Hydrided Zircaloy- 4 cladding tubes: an elastoplastic composite with anisotropic matrix and anisotropic distribution of inclusions}

\subsection{Microstructure of hydrided Zircaloy-4 cladding tubes}

The made up material of cladding tubes containing nuclear fuel is often a zirconium alloy. The useful properties and limitations of the as made standard Zircaloy- 4 were recalled in the introduction.

After the manufacturing process, zirconium based alloys structures contain about $10 \mathrm{ppm}$ of hydrogen (Northwood and Kosasih, 1983). During their life in reactors, these structures pick up some hydrogen from various sources: fuel humidity, hydrogen produced by water radiolysis, oxidation of the Zircaloy by the water coolant, etc. (Northwood and Kosasih, 1983; Aitchison, 1969). This picked-up hydrogen diffuses in the structure and precipitates over a given solubility limit, leading to some zirconium hydrides (Sawatzky, 1960). These hydrides can be found under three crystallographic forms: the $\gamma$-hydrides $(\mathrm{ZrH})$ and the $\epsilon$-hydrides $\left(\mathrm{ZrH}_{2}\right)$ with a face-centered tetragonal structure, and the $\delta$-hydrides $\left(\mathrm{ZrH}_{2}\right)$ with a face-centered cubic structure. If very high hydrogen concentrations can be locally observed without any adverse associated effects, the presence of small quantities of hydrides can drastically reduce both the overall ductility and the fracture toughness of the hydrided Zircaloy, as well at room temperature as in standard conditions reached in reactors (Kearns, 1967; Coleman and Hardie, 1966). Then, the associated embrittlement strongly depends on the morphology and on the orientation of the hydrides (Ells, 1968). The shape of these hydrides is often described as a 'cornflakes'-like shape (Perovic and Weatherly, 1984), and modelled by a parallelepipedic shape ('platelets') (Shi and Puls, 1994; Wäppling et al., 1997). Moreover, these hydrides are mainly oriented in an axial-circumferential plane and are randomly distributed in space (Arsène, 1997). Fig. 1 shows a portion of a cladding tube (gray parts are platelets of hydrides).

The effect of irradiation on Zircaloy properties is less clearly identified. One admits that the irradiation induces an overall hardening of the Zircaloy: increase of the yield stress and decrease of the ductility (see Fandeur, 2001 and references therein). However, the quantification of these effects often depends on how the so-called term 'irradiation' is interpreted. Concerning an in situ irradiation, both neutronic and hydrogenation effects have to be taken into account. This situation could be convenient for an empirical or semi-empirical description of cladding tubes understood as a complete structure, but do not lead to a proper mechanical description of the separate effects of the irradiation and hydrogenation on the Zircaloy material. Since a micromechanical approach is adopted in the present work, these effects have to be clearly distinguished. 'Irradiation' is thus here understood as the neutronic effects produced on each phase constituting the irradiated and hydrided Zircaloy.

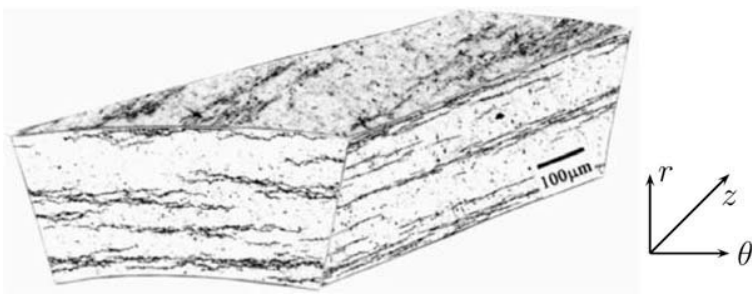

Fig. 1. Part of a hydrided cladding tube (Lee and Hong, 2002). 
After some years in a reactor, a cold worked stress-relieved Zircaloy-4 cladding tube exhibits five concentric zones: two inner oxide and hydride-fully dense layers, a central layer with hydride platelets and two outer hydride-fully dense and oxide layers. This work focuses on the mechanical behavior of the central zone, which occupies the largest part of the cladding tube (at least $80 \%$ of the radius). The investigated material is depicted in Fig. 2: hydrides form parallel platelets perpendicular to the radial direction and whose centers are isotropically distributed in space.

\subsection{Properties of the phases}

Following the experimental results of Delobelle et al. (1996), Kuroda et al. (2002), Yamanaka et al. (1999, 2001) and Desquines and Fédérici (2001), the studied two-phase metal-matrix composite is assumed to be made of an elastoplastic and anisotropic matrix and elastic inclusions. The matrix behavior is supposed to be isotropic in its elastic part and orthotropic in its plastic part, following the Hill criterion; and the inclusions are supposed to be linear elastic and isotropic. Only monotonic loading are considered here and the elastoplastic behavior for the matrix is replaced by a non-linear elastic behavior. The constitutive behavior of each phase of the composite is assumed to be governed by a potential $w^{(i)}$ such that the microscopic strain and stress fields $(\epsilon$ and $\sigma$ ) are related by:

$\sigma=\frac{\partial w^{(i)}}{\partial \epsilon}(\epsilon) \quad i=1,2$.

The dual potentials of $w^{(i)}$ are denoted $v^{(i)}$ such that:

$\epsilon=\frac{\partial v^{(i)}}{\partial \sigma}(\sigma) \quad i=1,2$.

The relations (1) and (2) are used under the small strains assumption. In the following equations, exponents 1 and 2 refer to the matrix and to the inclusions, respectively.

The stress potential related to the linear elastic and isotropic inclusions reads:

$v^{(2)}(\sigma)=v_{\text {ela }}^{(2)}(\sigma) \quad$ with $\quad v_{\text {ela }}^{(2)}(\sigma)=\frac{1}{2 k^{(2)}} \sigma_{m}^{2}+\frac{1}{6 \mu^{(2)}}\left(\sigma_{e q}^{\mathrm{VM}}\right)^{2}$,

and the stress potential related the elastoplastic and anisotropic matrix reads: $v^{(1)}(\sigma)=v_{\text {ela }}^{(1)}(\sigma)+v_{\text {pla }}^{(1)}(\sigma)$ with $v_{\text {ela }}^{(1)}(\sigma)=\frac{1}{2 k^{(1)}} \sigma_{m}^{2}+\frac{1}{6 \mu^{(1)}}\left(\sigma_{e q}^{\mathrm{VM}}\right)^{2}$,

where coefficients $k^{(i)}$ and $\mu^{(i)}$ denote respectively to the bulk and shear moduli of the phase $i(i=1,2)$, the quantity $\sigma_{e q}^{\mathrm{VM}}=\sqrt{\frac{3}{2}\left(\sigma^{d}: \sigma^{d}\right)}$ denotes the von Mises stress, $\sigma^{d}$ denotes the deviatoric part of $\sigma$, and $\sigma_{m}=\frac{1}{3} \operatorname{Tr}(\sigma)$ is the first invariant of the stress.

Following Mücke and Bernhardi (2003), the anisotropic plastic behavior of the matrix can be derived from the following stress potential, which states a multiaxial RambergOsgood law for anisotropic materials:

$$
\begin{aligned}
v_{p l a}^{(1)}(\sigma)= & \frac{\left(\sigma_{o}^{(1)}\right)^{2}}{6 \mu^{(1)}} \times H\left(\sigma_{e q}^{\mathrm{H}}-\sigma_{o}^{(1)}\right) \\
& \times\left[\frac{2}{n+1}\left(\left(\frac{\sigma_{e q}^{\mathrm{H}}}{\sigma_{o}^{(1)}}\right)^{n+1}-1\right)-\left(\left(\frac{\sigma_{e q}^{\mathrm{H}}}{\sigma_{o}^{(1)}}\right)^{2}-1\right)\right],
\end{aligned}
$$

where $H$ is Heavyside's function, the scalar $n$ is the hardening coefficient ( $n=1$ refers to the elastic case and $n \rightarrow+\infty$ refers to the perfectly plastic case), $\sigma_{o}^{(1)}$ denotes the elastic limit of the matrix, and the quantity

$\sigma_{e q}^{\mathrm{H}}=\sqrt{\frac{3}{2}\left(\sigma^{d}: \mathbb{M}: \sigma^{d}\right)}$

is the so-called Hill stress ( $\mathbb{M}$ being a fourth order tensor sometimes called matrix of anisotropy).

\section{A micromechanical model based on variational bounds}

\subsection{Formulation of the problem}

We consider a representative volume element (RVE) $V$ as depicted in Fig. 2. Each phase $i$ occupies a domain $V^{(i)}$, and its volume fraction $f^{(i)}$ is defined as $\left|V^{(i)}\right| /|V|$, where $|\mathscr{D}|$ denotes the volume of any domain $\mathscr{D}$. Moreover, the spatial averages of any quantity $A$ over the volume $V$ and over $V^{(i)}$ are respectively defined as :

$$
\langle A\rangle=\frac{1}{|V|} \int_{V} A d V \quad \text { and } \quad\langle A\rangle^{(i)}=\frac{1}{\left|V^{(i)}\right|} \int_{V^{(i)}} A d V .
$$

Considering a quasi-static problem and neglecting the body forces, the effective behavior is sought in the same form as for the phases (Eq. (1)):

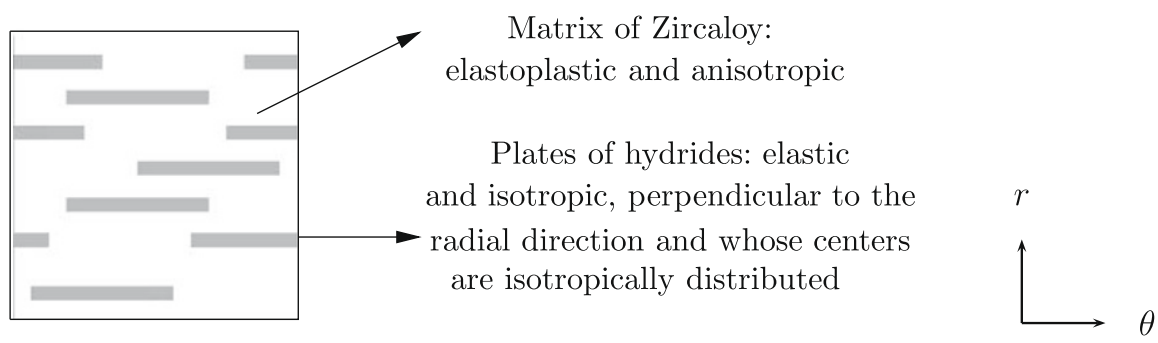

Fig. 2. The studied material. 
$E=\frac{\partial U^{\mathrm{eff}}(\Sigma)}{\partial \Sigma}$,

where $E$ and $\Sigma$ are the macroscopic strain and stress respectively:

$E=\langle\epsilon\rangle, \quad \Sigma=\langle\sigma\rangle$,

and $U^{\text {eff }}$ is the effective stress-energy potential, which satisfies:

$U^{\mathrm{eff}}(\Sigma)=\inf _{\tau \in \mathscr{S}(\Sigma)}\langle v(\tau)\rangle$,

where $v=v^{(i)}$ in each phase $i$, and $\mathscr{S}$ is the set of admissible stress-functions, which depends on the localization method. When homogeneous stress boundary conditions are considered, this set is defined as ( $v$ being the unit outer normal vector to the RVE):

$\mathscr{S}(\Sigma)=\{\tau, \operatorname{div}(\tau)=0$ in $V, \tau \cdot v=\Sigma \cdot v$ on $\partial V\}$,

Determining exactly this effective stress-energy potential is a formidable task, that cannot yet be achieved from a general point of view. In the next section, this effective stress-energy potential is lower bounded using the variational principle of Ponte Castañeda (1991) based on a linear comparison composite (LCC). The equivalence between this principle and the modified secant method of Suquet (1995) leads to solve a linear elastic homogenization problem for the LCC, whose elastic properties of phases derive from some secant moduli evaluated at the second moment of the stress.

3.2. The variational method of Ponte Castañeda (1991) and its link with the modified secant method of Suquet (1995)

Considering a linear comparison composite described by a field of compliance tensors $\mathbb{L}_{o}(x)$, and defining the following quantity $(\otimes$ denotes the tensor product):

$\mathbb{S}=\frac{1}{2}(\sigma \otimes \sigma)$,

the stress-energy potential $U^{\text {eff }}(\Sigma)$ can be rewritten as (Ponte Castañeda, 1991):

$U^{\text {eff }}(\Sigma)=\sup _{\mathbb{L}_{0}(x)}\left\{U_{o}^{\text {eff }}(\Sigma)-\left\langle\sup _{\mathbb{S}}\left\{\mathbb{L}_{o}(x):: \mathbb{S}-G(x, \mathbb{S})\right\}\right\rangle\right\}$,

where $U_{o}^{\text {eff }}(\Sigma)$ is the stress-energy potential of the LCC, and $G(x, S)=v(x, \sigma)$.

Considering now a LCC characterized by a set of piecewise constant compliances $\mathbb{L}_{0}^{(i)}$ (for instance, a two-phase LCC having the same microstructure as the considered non-linear composite), the optimization problem (13) becomes lower bounded by:

$U^{\mathrm{eff}}(\Sigma) \geqslant \bar{U}^{\mathrm{eff}}(\Sigma)=\sup _{\mathbb{L}_{0}^{(i)}}\left\{U_{0}^{\mathrm{eff}}(\Sigma)-\sum_{i=1}^{i=2} f^{(i)} \bigvee^{(i)}\left(\left(\mathbb{L}_{0}^{(i)}\right)^{-1}\right)\right\}$

with,

$V^{(\mathrm{i})}\left(\left(\mathbb{L}_{0}^{(\mathrm{i})}\right)^{-1}\right)=\sup _{\sigma}\left\{v_{0}^{(\mathrm{i})}(\sigma)-v^{(\mathrm{i})}(\sigma)\right\}$, where $v_{0}^{(i)}$ denotes the microscopic stress-energy potential of each phase $i$ of the LCC. This lower bound $\bar{U}^{\text {eff }}(\Sigma)$ turns now to be evaluated.

As pointed out by Ponte Castañeda and Suquet (1998), this variational procedure can be interpreted as a secant one, where the secant moduli of the phases are evaluated at the second moments of the fields. This shed light on why the "variational linear comparison method" is also known as the "modified secant method" (Suquet, 1995). Recalling that the chosen LCC has the same geometry as the non-linear composite (second order of the stress uniform in each phase), and that the inclusions are linear elastic, the use of this equivalence leads to the following system comprising a non-linear problem related to the matrix and a linear problem related to the LCC :

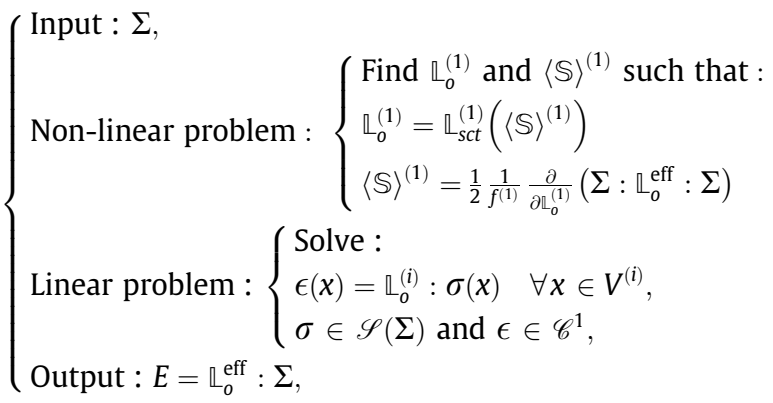

where $\langle S\rangle^{(1)}$ is the second moment of the stress on the matrix phase of the LCC (Ponte Castañeda and Suquet, 1998), $\mathbb{L}_{s c t}^{(i)}$ is the secant compliance of the phase $i$ of the LCC, defined by

$\epsilon=\frac{\partial v^{(i)}(\sigma)}{\partial \sigma}=\mathbb{L}_{s c t}^{(i)}(\sigma): \sigma$,

and $\mathbb{L}_{0}^{\text {eff }}$ is the effective compliance of the LCC, which is determined in the next section, giving:

$E=\frac{\partial U_{o}^{\text {eff }}(\Sigma)}{\partial \Sigma}=\mathbb{L}_{o}^{\text {eff }}(\Sigma): \Sigma$.

\subsection{The estimate for the overall behavior of the $L C C$}

Since the chosen LCC has the same microstructure as the non-linear composite, an estimate for the overall behavior of the LCC has to take into account separately the shape and the distribution of the inclusions. The estimate of Ponte Castañeda and Willis (1995) is thus here retained:

$\mathbb{L}_{0}^{\text {eff }}=\mathbb{L}_{0}^{(1)}+f^{(2)}\left(\left(\mathbb{L}^{(2)}-\mathbb{L}_{0}^{(1)}\right)^{-1}+\left(1-f^{(2)}\right) \mathbb{Q}\right)^{-1}$,

where:

$\mathbb{Q}=\left(\mathbb{L}_{0}^{(1)}\right)^{-1}-\frac{1}{1-f^{(2)}}\left(\mathbb{L}_{0}^{(1)}\right)^{-1}:\left(\mathbb{P}_{i}-f^{(2)} \mathbb{P}_{d}\right):\left(\mathbb{L}_{0}^{(1)}\right)^{-1}$.

The fourth order tensors $\mathbb{P}_{i}$ and $\mathbb{P}_{d}$ are the Hill tensors related respectively to the shape and to the distribution of the inclusions. In this estimate, the inclusions are represented by aligned and isotropically distributed ellipsoids, 
defined by two aspect ratios: $w_{L}$, the ratio of the width in the radial direction $(r)$ to the length in the longitudinal direction $(z)$ of the ellipsoids, and $w_{T}$, the ratio of the width in the radial direction $(r)$ to the length in the transverse direction $(\theta)$ of the ellipsoids. The link between these two aspect ratios and those of the associated platelets is examinated in Section 4.3. Moreover, this estimate states that the reference medium is the matrix phase, which is here anisotropic. The tensor $\mathbb{P}_{i}$ thus refers to the Hill tensor concerning the Eshelby problem of an ellipsoidal inclusion in an unbounded and anisotropic medium. Although recent works allow to derive explicit expressions (but rather complex in the present context) of the Hill tensor in this case (Masson, 2008), one assumes that the tensor $\mathbb{P}_{i}$ is wellestimated when replacing the matrix behavior in this Eshelby problem by its projection onto the isotropic basis:

$P_{\sqrt{K}}\left(\mathbb{L}_{o}^{(1)}\right)=\frac{\mathbb{Q}_{o}^{(1)}:: \mathbb{\rrbracket}}{\mathbb{J}:: \mathbb{J}} \mathbb{\mathbb { W }}+\frac{\mathbb{L}_{o}^{(1)}:: \mathbb{K}}{\mathbb{K}:: \mathbb{K}} \mathbb{K}$,

where $\rrbracket_{i j k l}=1 / 3 \delta_{i j} \delta_{k l}$ and $\mathbb{K}=\rrbracket-\rrbracket\left(\right.$ with $\rrbracket_{i j k l}=1 / 2\left(\delta_{i k} \delta_{j l}+\right.$ $\delta_{i l} \delta_{j k}$ ) and $\delta$ the Kronecker symbol). The same assumption is used for the Hill tensor $P_{d}$ related to the Eshelby problem of a spherical inclusion (statistically isotropic distribution). The relevance of this assumption is checked in the case of flat and circular inclusions $\left(w_{T}=w_{L}=0\right)$ in an orthotropic medium, for which Suvorov and Dvorak (2002) give an analytical solution of the Hill tensor: the two Eshelby tensors obtained with the assumption (21) and with the exact solution of Suvorov and Dvorak (2002), respectively, are numerically very close to each other in the context of the studied problem. It is assumed in the sequel that this hypothesis is still valid for the LCC and for any aspect ratios $0 \leqslant w_{L}, w_{T} \leqslant 1$. The expressions of these Hill tensors $\mathbb{P}_{i}$ and $\mathbb{P}_{d}$ can be derived from the corresponding Eshelby tensors whose expressions are given in Mura (1982).

Moreover, the effective compliance (19) is positive definite under conditions on the distribution type, the inclusions shape and the volume fractions of the phases. For a spherical distribution, when $w_{L}=w_{T}=w$, the condition $f^{(2)} \leqslant w$ ensures the consistency of (19) by introducing "security ellipsoids" surrounding the inclusions (Ponte Castañeda and Willis, 1995). From a physical point of view, this condition consists in excluding particule overlap.

\section{Implementation of the model and identification of the morphological parameter}

Keeping in mind that the practical use of the present model concerns the particular case of a Zircaloy- 4 matrix (in a cold worked stress state) containing hydride inclusions, the validation of the model is here performed with mechanical data related to this material. This validation is done in three steps. First, material properties of the Zircaloy- 4 are given. Second, the numerical implementation of the model is detailed. Third, the morphological parameters of the model are identified and the model is validated by comparisons with three-dimensional periodic finite element simulations. Finally, some predictions and abilities of the model are presented; in particular, the role of a new parameter introduced in the sequel allowing a smooth transition from a Hill plastic matrix to a von Mises one is fully detailed ("Hill-to-von Mises" parameter).

\subsection{Material properties}

The values of the material properties used for Zircaloy-4 in the cold worked stress state and for the inclusions of hydrides at room temperature are given in Table 1. Elastic coefficients of the matrix are given by Desquines and Fédérici (2001), whereas the ones of the inclusions are given by Yamanaka et al. (1999) and Yamanaka et al. (2001). The value of the initial yield stress of the matrix in the $z$ direction is given by Delobelle et al. (1996).

Values of matrix $\mathbb{M}$ components are extracted from the experimental data of Delobelle et al. (1996) on non-irradiated and hydrided Zircaloy-4 in the cold worked stress state. Since coefficients $\mathbb{M}_{r z r z}$ and $\mathbb{M}_{r \theta r \theta}$ are difficult to be experimentally determined, they are here arbitrarily fixed to 1 . These coefficients have a minor influence when the applied load is mainly longitudinal $(z)$ and transverse $(\theta)$ : this is precisely the case during a Reactivity Initiated Accident, which is the main motivation of this study. Using the following vectorial representation of $\sigma$ :

$\sigma=\left\{\sigma_{r r}, \sigma_{\theta \theta}, \sigma_{z z}, \sqrt{2} \sigma_{\theta z}, \sqrt{2} \sigma_{r z}, \sqrt{2} \sigma_{r \theta}\right\}^{T}$,

the matrix $\mathbb{M}$ takes thus the form:

$\mathbb{M}=\left(\begin{array}{llllll}1.0 & -0.72 & -0.28 & 0 & 0 & 0 \\ -0.72 & 1.1 & -0.38 & 0 & 0 & 0 \\ -0.28 & -0.38 & 0.66 & 0 & 0 & 0 \\ 0 & 0 & 0 & 1.7 & 0 & 0 \\ 0 & 0 & 0 & 0 & 1 & 0 \\ 0 & 0 & 0 & 0 & 0 & 1\end{array}\right)$.

These anisotropy coefficients are consistent with the recent work of Leclercq et al. (2007).

Anticipating on the last part of the paper (Section 5) and following some authors as Nakatsuka and Nagai (1987), one suspects that irradiation effects induce a progressive decrease of the plastic anisotropy of the Zircaloy matrix. A slightly modified anisotropic matrix $\widetilde{\mathbb{M}}$ is substituted to $\mathbb{M}$ in the Hill criterion:

$\widetilde{\mathbb{M}}=(1-\alpha) \mathbb{K}+\alpha \mathbb{M}$

The parameter $\alpha$ allows a smooth transition from a Hill criterion $(\alpha=1)$ to a standard von Mises one $(\alpha=0)$. A new definition of the Hill stress is thus used:

\section{Table 1}

Material properties used for the Zircaloy-4 in the cold worked stress state and for the inclusions of hydrides at room temperature after Desquines and Fédérici (2001), Yamanaka et al. (1999), Yamanaka et al. (2001) and Delobelle et al. (1996).

\begin{tabular}{lll}
\hline & Matrix & Inclusions \\
\hline Young's modulus & $98 \mathrm{GPa}$ & $135 \mathrm{GPa}$ \\
Poisson's ratio & 0.325 & 0.32 \\
Elastic limit & $500 \mathrm{MPa}$ & - \\
Hardening exponent $n$ & 71 & - \\
\hline
\end{tabular}


$\sigma_{e q}^{\mathrm{H}}=\sqrt{\frac{3}{2} \sigma^{d}: \widetilde{\mathbb{M}}: \sigma^{d}}$.

This "Hill-to-von Mises" parameter $\alpha$ is a key concept of the paper. When $\alpha=1$, Eq. (24) reduces to $\widetilde{\mathbb{M}}=\mathbb{M}$ and the equivalent stress (25) corresponds to the initial Hill criterion: the matrix exhibits the same anisotropic plasticity as the one measured by Delobelle et al. (1996). In the following, this situation corresponds to a 'fresh' state, where the matrix does not undergo any irradiation. When $\alpha=0$, Eq. (24) reduces to $\widetilde{\mathbb{M}}=\mathbb{K}$ and the corresponding equivalent stress (25) reads $\sqrt{\frac{3}{2} \sigma^{d}: \mathbb{K}: \sigma^{d}}=\sqrt{\frac{3}{2} \sigma^{d}: \sigma^{d}}=\sigma_{e q}^{\mathrm{vM}}$, which corresponds to the von Mises criterion: the matrix exhibits in that case a standard isotropic plasticity. The parameter $\alpha$ allows thus a progressive transition from an initial anisotropic plasticity to an isotropic one. When $0<\alpha<1$, the situation is intermediate between a Hill criterion and a von Mises one. The effect of this parameter on the overall yield surface of the composite will be quantitatively characterized in due time.

\subsection{Implementation of the model}

The presented model is applied to the constitutive behaviors (2)-(6) of the considered composite.

The secant modulus of the matrix $\mathbb{L}_{s c t}^{(1)}$ is obtained by using Eqs. (2) and (17) for $i=1$ :

$\epsilon=\frac{\partial v^{(1)}(\sigma)}{\partial \sigma}=\mathbb{L}_{s c t}^{(1)}(\sigma): \sigma$.

Note that:

$\frac{\partial \sigma_{e q}^{\mathrm{H}}}{\partial \sigma}=\frac{3}{2 \sigma_{e q}^{\mathrm{H}}} \widetilde{\mathbb{M}}: \sigma^{d}$ and $\sigma^{d}=\mathbb{K}: \sigma$.

Then, using the stress-energy potential (4) with Eq. (5), we get:

$\mathbb{L}_{s c t}^{(1)}(\sigma)=\mathbb{L}_{e l}^{(1)}+\frac{1}{2 \mu^{(1)}}\left(\left(\left(\frac{\sigma_{e q}^{\mathrm{H}}}{\sigma_{o}^{(1)}}\right)^{n-1}-1\right) \widetilde{\mathbb{M}}: \mathbb{K}\right) H\left(\sigma_{e q}^{\mathrm{H}}-\sigma_{o}^{(1)}\right)$,

where $\mathbb{L}_{e l}^{(1)}$ denotes the compliance for the elastic linear and isotropic part of the matrix.

At this stage, it is worth noting that $\mathbb{L}_{e l}^{(1)}$ is written in terms of the von Mises stress $\sigma_{e q}^{\mathrm{VM}}$ (see Eq. (4)) and that the right hand side of Eq. (28) is written in terms of the Hill stress $\sigma_{e q}^{\mathrm{H}}$. This particularity, due to the matrix anisotropy, does not allow to express the strain-energy potential related to this phase in a simple manner. Our stress approach, scarcely employed for (perfect) plastic behaviors, provides an original and efficient mean to circumvent to this issue.

The tensor $\mathbb{L}_{s c t}^{(1)}$ can be written in terms of $\mathbb{S}$, noting that (see Eqs. (12), (25) and (27) and recalling that $\widetilde{\mathbb{M}}$ is assumed constant by phase):

$\sigma_{e q}^{\mathrm{H}}=\sqrt{3 \widetilde{\mathbb{M}}::(\mathbb{K}: \mathbb{S}: \mathbb{K})}$.

The partial derivative of $\Sigma: \mathbb{L}_{o}^{\text {eff }}: \Sigma$ with respect to $\mathbb{L}_{o}^{(1)}$ used in the problem (16) is estimated component by component $(i, j, k, l=1,2,3)$ :

$$
\begin{aligned}
\left.\frac{\partial \Sigma: \mathbb{L}_{o}^{\text {eff }}\left(\mathbb{L}_{o}^{(1)}, \chi\right): \Sigma}{\partial \mathbb{L}_{0}^{(1)}}\right)_{i j k l} & =\frac{\partial \Sigma: \mathbb{L}_{o}^{\text {eff }}\left(\mathbb{L}_{o}^{(1)}, \chi\right): \Sigma}{\partial \mathbb{L}_{o i j k l}^{(1)}} \\
& \approx \frac{\Sigma: \mathbb{L}_{o}^{\text {eff }}\left(\mathbb{L}_{o}^{(1)}+h \mathbb{U}, \chi\right): \Sigma-\Sigma: \mathbb{L}_{o}^{\mathrm{eff}}\left(\mathbb{L}_{o}^{(1)}, \chi\right): \Sigma}{h} \\
& =\mathbb{D}\left[\Sigma: \mathbb{L}_{o}^{\mathrm{eff}}: \Sigma\right]_{i j k l} \text { (notation) }
\end{aligned}
$$

where $\chi$ states for the set of variables $\left(\mathbb{L}^{(2)}, w_{L}, w_{T}, f^{(2)}\right), \mathbb{H}$ is a unit fourth order tensor which has the major and the minor symmetries, linked to the gradient component (only the components $i j k l, k l i j, j i k l, i j l k, j i l k$ are non-zero). The value of the scalar $h$ is chosen small enough to have the same derivative by upper value $(+h)$ or by lower value $(-h)$ (in the following, $h=10^{-6}$ ). Under this condition, the incremental quotient method (30) gives accurate results with material properties given in Table 1 and does not suffer from a strong sensitivity to parameter $h$.

Using Eqs. (16), (29) and (30) evaluated at $\mathbb{L}_{o}^{(1)}=\mathbb{L}_{s c t}^{(1)}$ $\left(\sigma_{e q}^{\mathrm{H}}\right)$, the final problem can be rewritten in the form of a non-linear scalar equation with respect to the Hill stress:

$$
\left(\sigma_{e q}^{\mathrm{H}}\right)^{2}=3 \widetilde{\mathbb{M}}::\left[\mathbb{K}:\left[\frac{1}{2 f^{(1)}} \mathbb{D}\left[\Sigma: \mathbb{L}_{o}^{\text {eff }}: \Sigma\right]\left(\mathbb{L}_{\text {sct }}^{(1)}\left(\sigma_{e q}^{\mathrm{H}}\right)\right)\right]: \mathbb{K}\right] .
$$

This equation is solved using a Newton-Raphson algorithm with numerical jacobian: about 15 iterations are needed to obtain a precision of $10^{-6}$ on the function which has to be nullified in Eq. (31).

\subsection{Numerical identification of the morphological parameters and validation of the model}

As already mentioned, the proposed model is based on a LCC containing ellipsoidal inclusions, with a longitudinal and transversal aspect ratios denoted by $w_{L}$ and $w_{T}$, whereas the inclusions in the real material are akin to platelets (aspect ratios $w_{L}^{p}$ and $w_{T}^{p}$ ). Comparison with numerical simulation results enables us to identify the link between the ellipsoidal and platelet aspect ratios. This identification is performed in the simple case where $w_{L}=w_{T} \equiv w$ and $w_{L}^{p}=w_{T}^{p} \equiv w^{p}$.

Assuming that the medium is periodic, the finite element software Abaqus ${ }^{\circledR}$ is used to carry out simulations on unit cells containing parallel and randomly distributed square base platelets (see Fig. 3). Only constant aspect ratio unit cells are considered. We examine three cases and set $w^{p}$ to $0.05,0.1$ and 0.125 (one sample for each $w^{p}$ ). The unit cells are submitted to macroscopic strain rate $\dot{E}$ and periodic boundary conditions are introduced to ensure the periodicity of the microscopic strain and stress (Michel et al., 2001). The inclusions are elastic (Young's Modulus and Poisson ratio given in Table 1). The matrix is elastic plastic with an isotropic elastic part and an orthotropic plastic part with an isotropic exponential hardening (material parameters given in Table 1 and Eq. (23)). The Hill-to-von Mises parameter $\alpha$ is set to 1 .

The identification of the morphological parameter of the model $w$ is performed for uniaxial tension tests in the $z$ direction and in the $\theta$ direction in the case of thin platelets $\left(w^{p}=0.05\right.$ and $\left.f^{(2)} \approx 4.4 \%\right)$. This identification is per- 


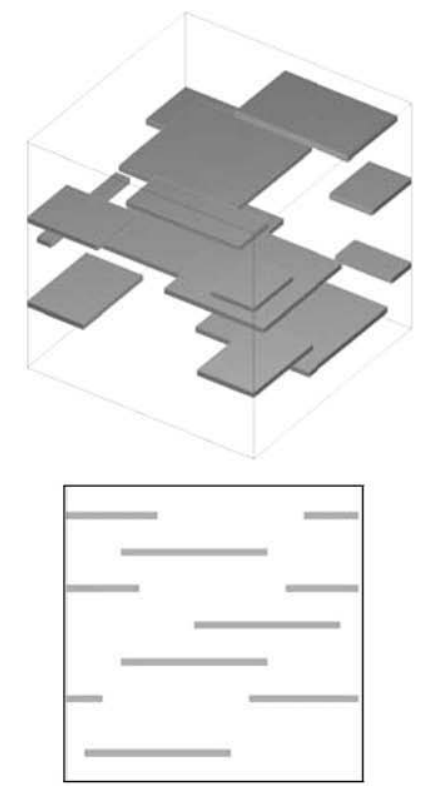

$w^{p}=0.05$
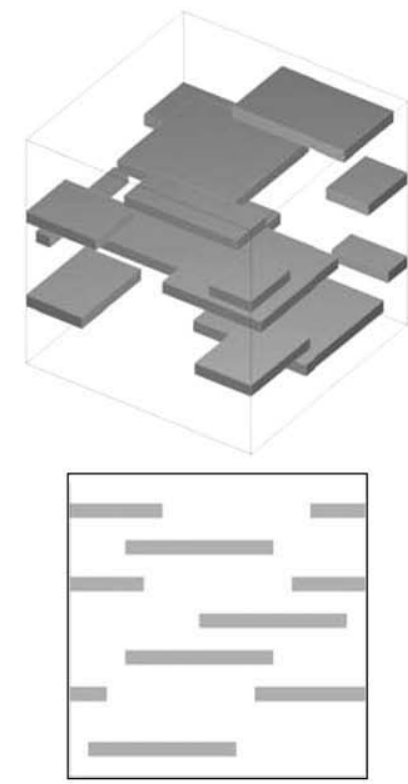

$w^{p}=0.1$
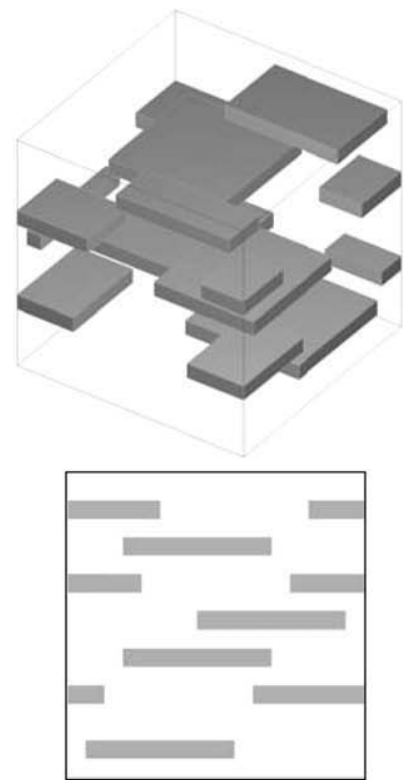

$w^{p}=0.125$

Fig. 3. Various three-dimensional unit cells used for the numerical simulations and their projections onto the $r-\theta$ plane.

formed assuming that ellipsoidal aspect ratio $w$ and platelet aspect ratio $w^{p}$ are proportional. The accuracy of this identification is illustrated in Fig. 4. In the sequel, the following relation is used:

$w \approx 1.7 w^{p}$,

and the corresponding morphology for the platelets and for the ellipsoids onto the $r-\theta$ plane are shown in Fig. 5.

The model is validated for a less dilute case $w^{p}=0.125$, $f^{(2)} \approx 10.93 \%$. Fig. 6 shows the comparison between numerical periodic simulations and predictions of the model. Uniaxial tension tests in the $z$ direction and in the $\theta$ direction are considered, and the aspect ratios of the ellipsoids of the LCC invoked in the model depend on the aspect ratios of the platelets using the identified relation (32). In the sequel, this relation is assumed to be valid even when two different longitudinal and transversal aspect ratios are considered: $w_{T} \approx 1.7 w_{T}^{p}$ and $w_{L} \approx 1.7 w_{L}^{p}$.

\subsection{Predictions and abilities of the model}

In the elastic part, the overall anisotropy of the composite rises only from the shape and orientation of the inclu-
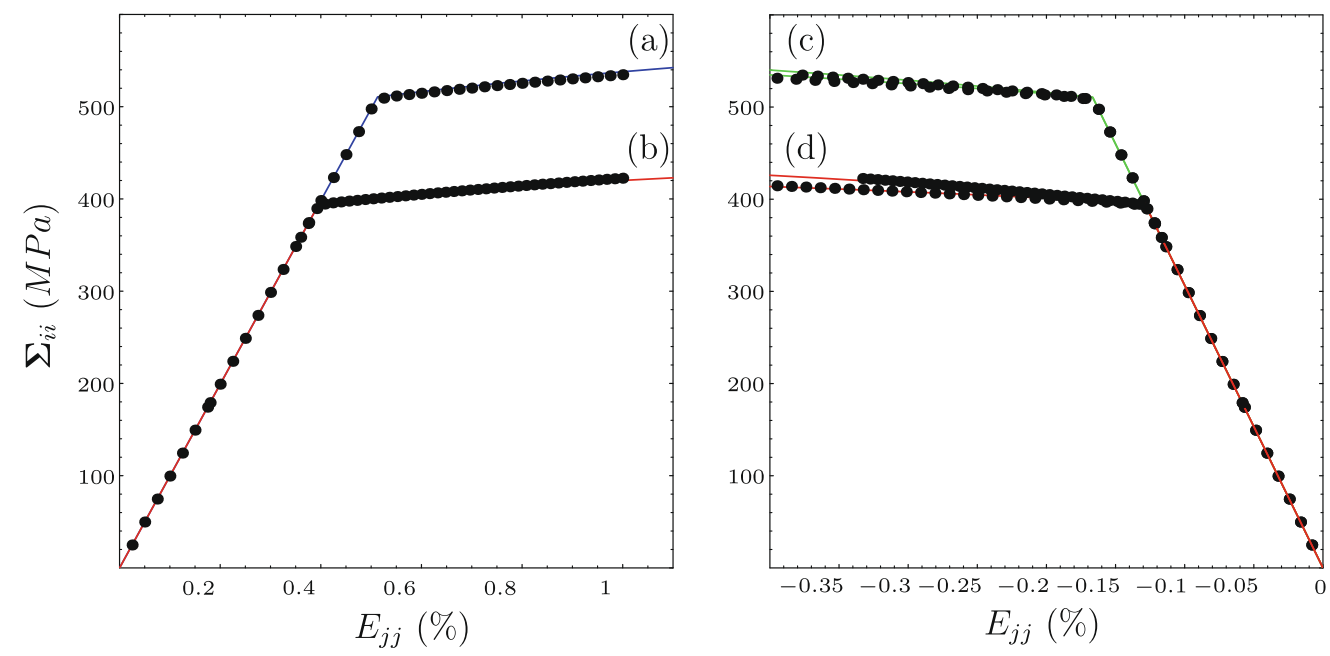

Fig. 4. Identification of the morphological parameter of the model $w$ (lines) by comparison with numerical simulations (points) for $f^{(2)} \approx 4.4 \%$ and $w^{p}=0.05$. Uniaxial tension test in the $z$ direction: (a) $\Sigma_{z z}$ vs. $E_{z z}$, (c) $\Sigma_{z z}$ vs. $E_{\theta \theta}$ and $E_{r r}$; and in the $\theta$ direction: (b) $\Sigma_{\theta \theta}$ vs. $E_{\theta \theta}$, (d) $\Sigma_{\theta \theta}$ vs. $E_{r r}$ and $E_{z z}$. The proposed model coincides with the numerical simulations for $w=0.088 \simeq 1.7 w^{p}$. 


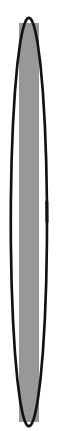

(a)

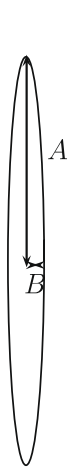

(b)



(c)
Fig. 5. Link between the ellipsoidal aspect ratio $w$ and the platelet aspect ratio $w^{p}$ in the transverse direction: (a) projection of a platelet and the associated ellipsoid $w_{T}=1.7 w_{T}^{p}$ onto the $r-\theta$ plane, (b) definition of $w_{T}$, and (c) definition of $w_{T}^{p}$.

sions (isotropic elasticity for the phases). This overall elastic anisotropy is quantified using a classical criterion of isotropy:

$\zeta(\mathbb{B})=1-\frac{\left\|\mathbb{B}-P_{ป \mathbb{K}}(\mathbb{B})\right\|}{\|\mathbb{B}\|}$,

where $\|\mathbb{B}\|=\sqrt{\mathbb{B}:: \mathbb{B}}$ for any fourth order tensor $\mathbb{B}$. The evolution of this criterion with the aspect ratio $w$ and the volume fraction of the inclusions $f^{(2)}$ is given in Fig. 7. As expected, and due to the statistically isotropic distribution in space of the inclusions, the elastic response of the composite exhibits an overall isotropy for any volume fraction when inclusions are spheroids $(w=1)$. For oblate inclusions $(w<1)$, the anisotropy of the overall elastic behavior increases with respect to volume fraction and decreases with respect to aspect ratio.

The overall anisotropy of the composite in its plastic part is characterized by three parameters in the model: the coefficient $\alpha$ of 'Hill-to-von Mises' criterion of the matrix (see Eq. (24)), the aspect ratio $w$ and the volume frac- tion of inclusions $f^{(2)}$. The separate effects of these parameters on the overall yield surface are illustrated in Fig. 8. As shown in Fig. 8 (top), the effect of the 'Hill-tovon Mises' parameter $\alpha$ ensures a smooth transition from a Hill criterion $(\alpha=1)$ to a von Mises one $(\alpha=0)$. As a general rule, Fig. 8 (top and bottom) illustrates the fact that the presence of elastic inclusions in a plastic matrix leads to increase the initial elastic domain. The size of this domain increases with respect to the volume fraction of inclusions $f^{(2)}$, and decreases with respect to the aspect ratio $w$. In particular, the present model quantifies the fact that thin inclusions aligned with a plane perpendicular to the $r$ direction significantly increase the size of the elastic domain in $z$ and $\theta$ directions but not in the $r$ direction (parallel assembly versus serie assembly).

Details of the influence of volume fraction and aspect ratio of inclusions on the plastic flow are given in Fig. 9,

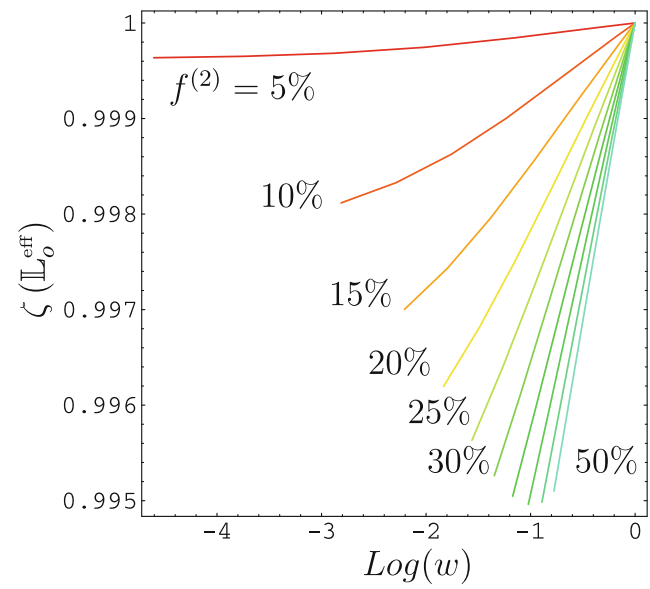

Fig. 7. Overall anisotropy of the elastic response versus shape of the inclusions $w$ for various volume fraction $f^{(2)}\left(f^{(2)}\right.$ varies from $5 \%$ to $50 \%$, the condition of 'security ellipsoids' $f^{(2)} \leqslant w$ is satisfied): for spherical inclusions $(w=1)$, the composite is isotropic; for oblate inclusions $(w<1)$, the higher the volume fraction (or the lower the aspect ratio) the higher the elastic anisotropy.
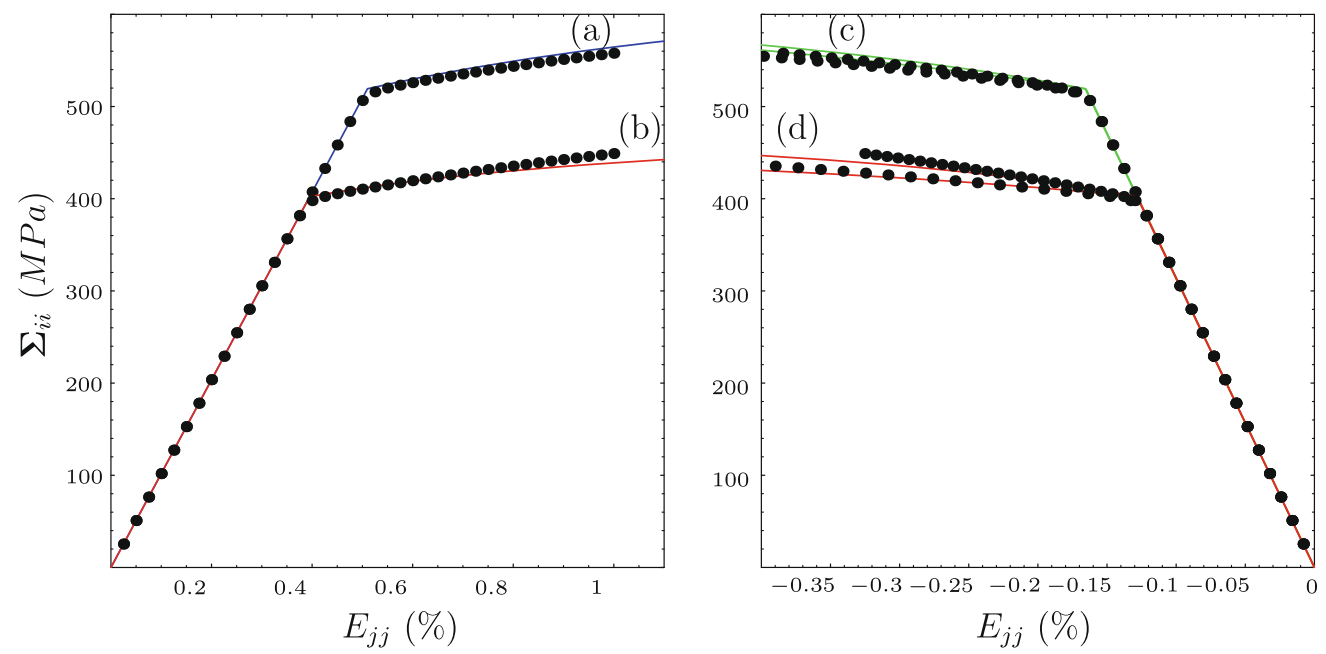

Fig. 6. Validation of the model. Same legend as Fig. 4 with $w^{p}=0.125, f^{(2)} \approx 10.93 \%$ and $w=1.7 w^{p}=0.221$. 
and the overall plastic anisotropy is compared for the three main directions $(r, \theta$ and $z)$ in Fig. 10. As depicted in these figures, the inclusions have a greater effect on plastic hardening than on initial yield surface. The magnitude of the yield stress and plastic hardening and their sensitivity to
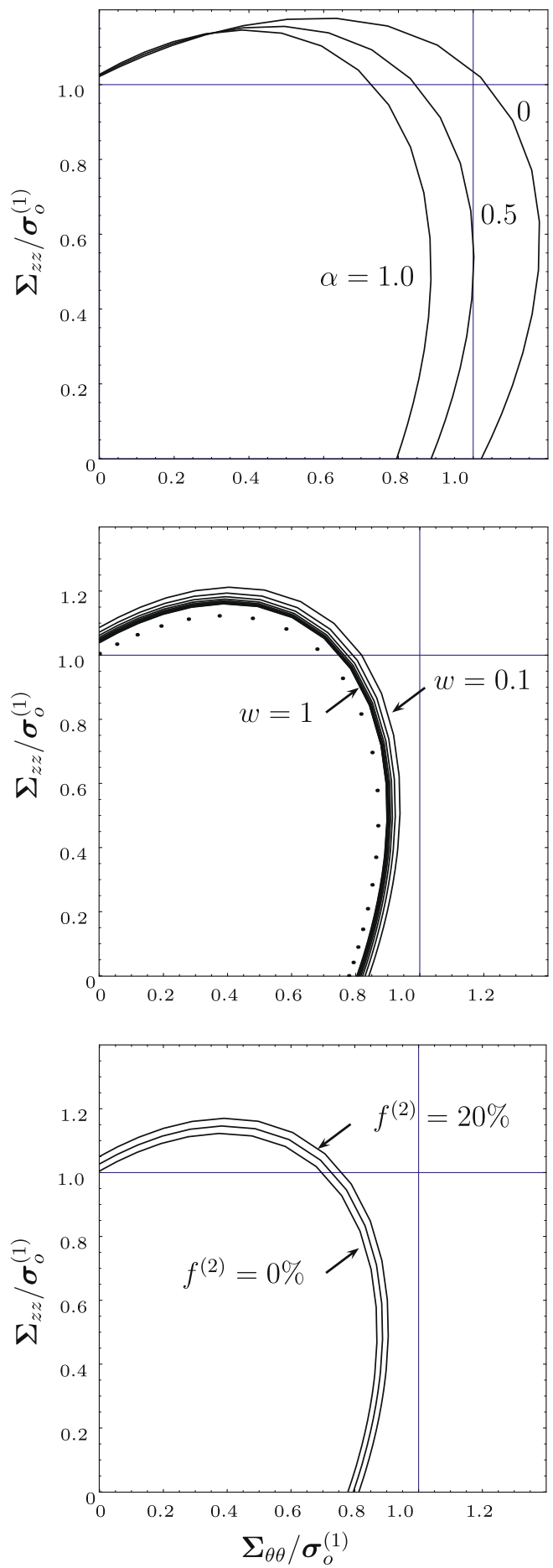

volume fraction and to aspect ratio are larger in $z$ direction than in $r$ or $\theta$ directions.

This model is applied next to the prediction of the plastic and anisotropic behavior of hydrided and irradiated Zircaloy.
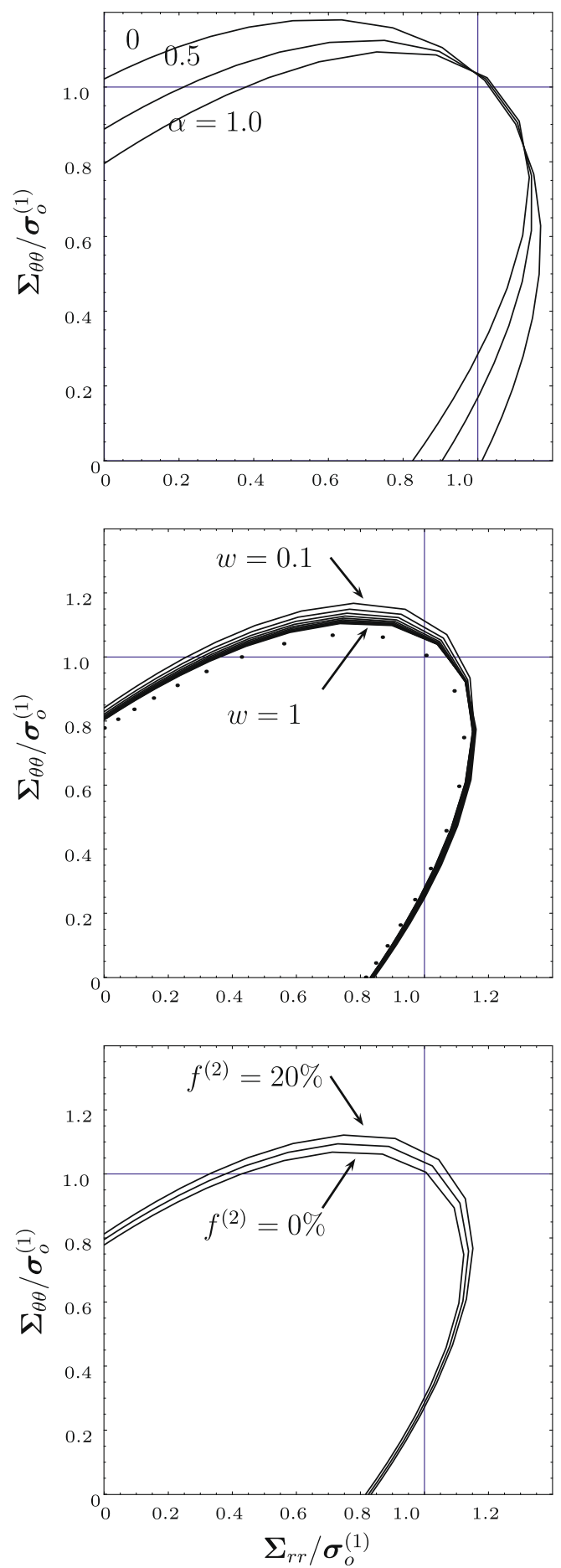

Fig. 8. Effect of the three parameters of the model on the anisotropy of the normalized initial effective yield surface: (top) effect of the 'Hill-to-von Mises' parameter $\alpha\left(w=0.5\right.$ and $\left.f^{(2)}=10 \%\right)$; (middle) effect of the inclusion aspect ratio $w\left(f^{(2)}=20 \%, \alpha=1\right.$ and $w$ varies from 1 to 0.1 ); (bottom) effect of the volume fraction of inclusions $f^{(2)}\left(w=0.5, \alpha=1\right.$ and $\left.f^{(2)}=0,10 \%, 20 \%\right)$. Points: normalized initial yield surface of the matrix without inclusion. 


\section{Application to the hydrided and irradiated Zircaloy}

This section is devoted to some applications of the proposed model to hydrided and irradiated Zircaloy. Material parameters of constituting phases have been given in Section 4. Model predictions are compared to
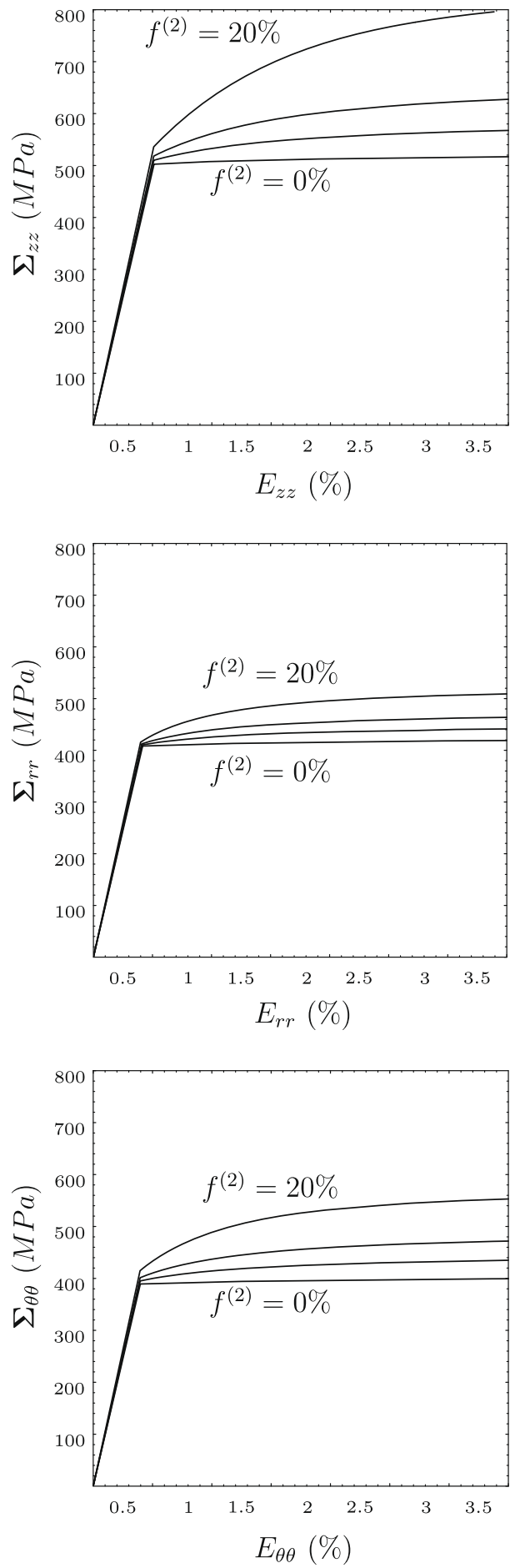

experimental data. We pay a particular attention to overall engineering parameters such as ultimate tensile stress and elongation in the longitudinal $(z)$ and transverse $(\theta)$ directions. Variations of plastic anisotropy due to hydriding and to irradiation respectively are studied and their combined effects are analyzed. We attempt to link the
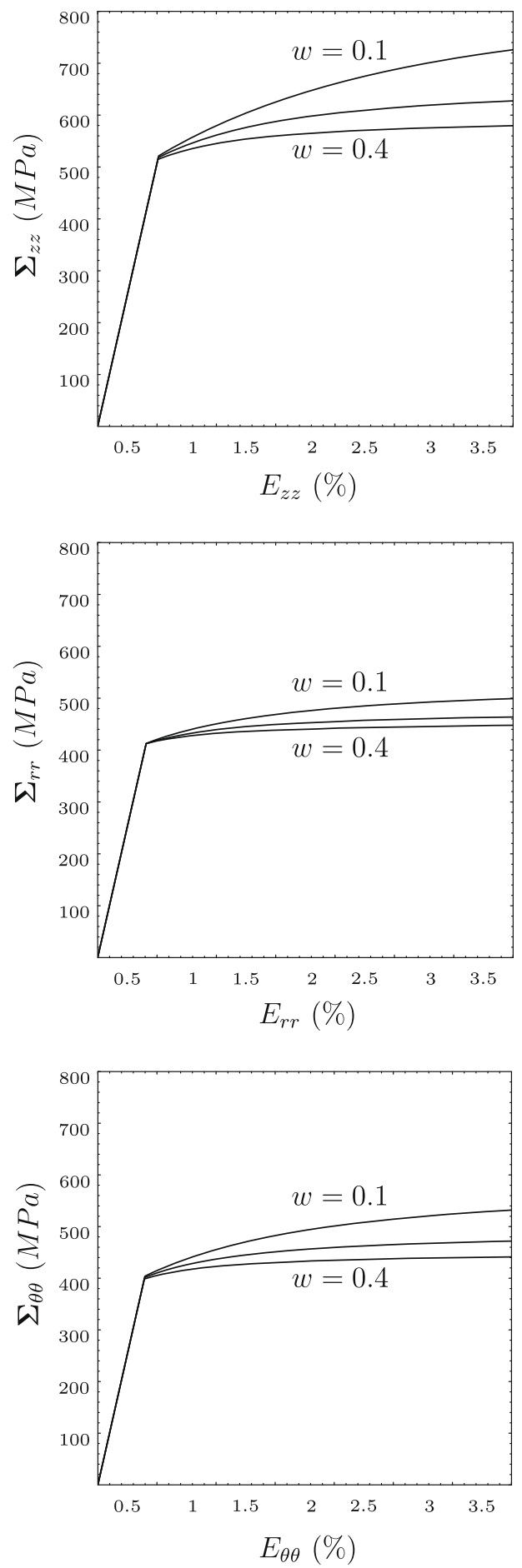

Fig. 9. Effect of the volume fraction $f^{(2)}$ (left column) and of the aspect ratio $w$ of inclusions (right column) on the plastic flow during uniaxial tension tests in the three directions successively: (left column) $w=0.2, \alpha=1$ and $f^{(2)}=0 \%, 0.05 \%, 10 \%, 20 \%$; (right column) $f^{(2)}=10 \%, \alpha=1$ and $w=0.1,0.2,0.4$. 


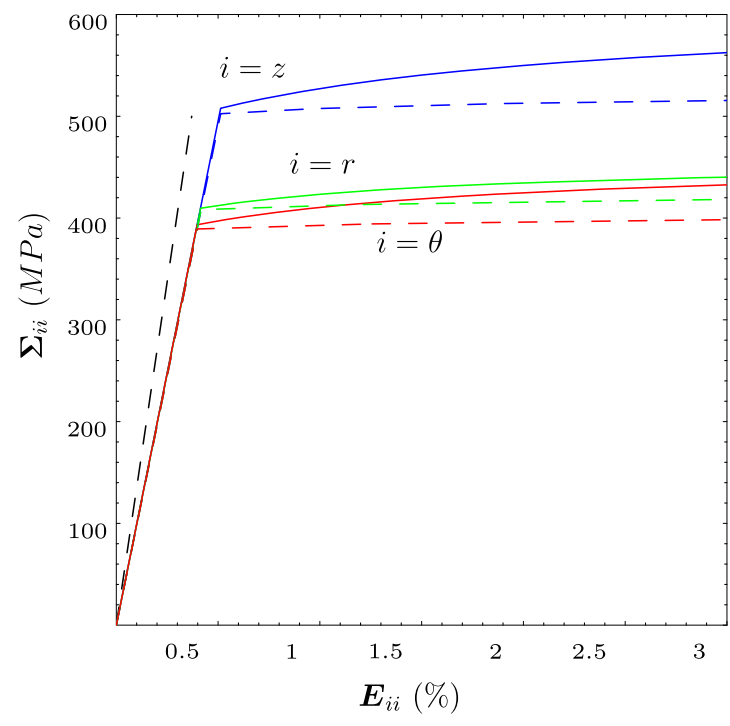

Fig. 10. Anisotropy of the plastic flow : comparison of the overall response of the composite during simple tension tests in the three directions successively $(i=r, \theta, z)$ for $w=0.1$ and $f^{(2)}=3 \%$. The yield stress and the plastic hardening increase from direction $\theta$ to direction $r$, and from direction $r$ to direction $z$. The dotted lines recall the uniaxial response in the three directions of the plastic matrix and elastic inclusions.

parameters of both phenomena (volume fraction $f^{(2)}$ of inclusion for the hydriding, and Hill-to-von Mises parameter $\alpha$ for the irradiation) to an integrated measure of the in-pile duration (the 'burn-up'), and a prediction of the overall plastic anisotropy with respect to the burn-up is compared to experimental data.

In what follows, the predictions of the proposed micromechanical-based model are compared to experimental data on the basis of two main overall engineering parameters that can be easily achieved by experimental techniques, namely the ultimate tensile stress, denoted by $\mathscr{R}$, and the uniform elongation, denoted by $\mathscr{A}$. The ultimate tensile stress $\mathscr{R}$ is simply defined as the peak load on an engineering stress-strain curve, and the uniform elongation $\mathscr{A}$ is defined as the plastic engineering strain associated to this ultimate tensile stress $\mathscr{R}$. In order to avoid any ambiguity, we underline that the present definition of the ultimate tensile stress $\mathscr{R}$ does not correspond to any localization process: the proposed model exhibits only strain hardening in a rational stress-strain diagram; a peak load can only be observed in an engineering diagram (see Fig. 11). This last value states here for the ultimate tensile stress. Since the applied part of the present study is devoted to the mechanical behavior of Zircaloy cladding tubes, the relevant parameters $\mathscr{R}$ and $\mathscr{A}$ concern the longitudinal $(z)$ and transverse $(\theta)$ directions, respectively denoted by $\mathscr{R}_{L}$ and $\mathscr{A}_{L}$ (longitudinal), and by $\mathscr{R}_{T}$ and $\mathscr{A}_{T}$ (transverse). The transverse-to-longitudinal anisotropy of Zircaloy tubes can be characterized by ratios $\mathscr{R}_{L} / \mathscr{R}_{T}$ and $\mathscr{A}_{L} / \mathscr{A}_{T}$. These ratios are plotted in Fig. 11 for the 'fresh' Zircaloy, i.e. without any hydride inclusions $\left(f^{(2)}=0\right)$, nor irradiation effects $(\alpha=1)$; the plastic anisotropy is fully defined by the Hill matrix (23).

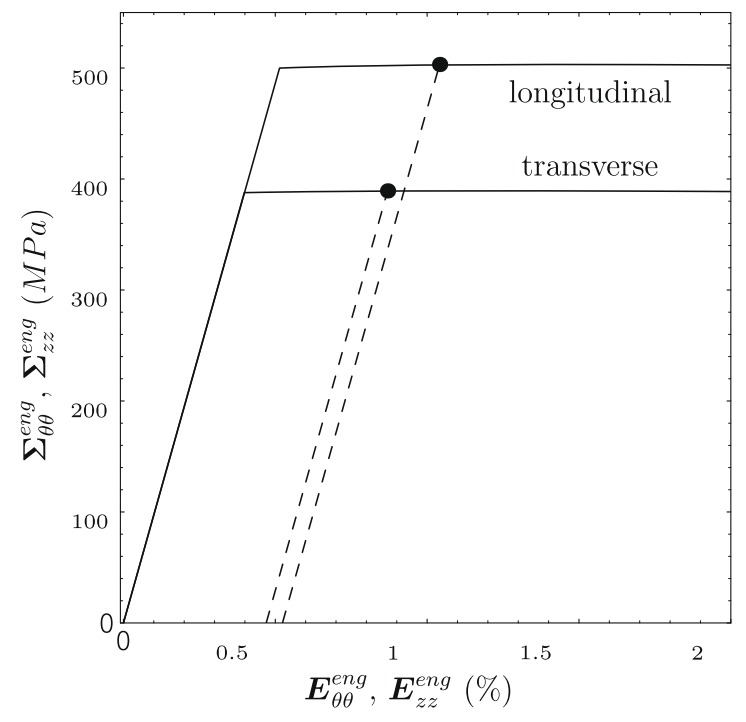

Fig. 11. Engineering stress-strain curves in the transverse and longitudinal directions. Initial anisotropy of the non hydrided, non irradiated Zircaloy $\left(\alpha=1, f^{(2)}=0\right)$. Transverse-to-longitudinal anisotropy: $\mathscr{R}_{T} / \mathscr{R}_{L}=0.77$ and $\mathscr{A}_{T} / \mathscr{A}_{L}=0.83$.

\subsection{Transverse behavior}

The initial anisotropy evolves with the presence of aligned inclusions. Fig. 12 compares the evolution of the normalized transverse ultimate tensile stress $\mathscr{R}_{T}$ with respect to the hydrogen content in the cladding to experimental data of Kuroda et al. (2002) and Grange et al. (2000). In this graph, $\mathscr{R}_{T}$ is normalized by the transverse ultimate tensile stress of non hydrided and non-irradiated Zircaloy, and the hydrogen content $[\mathrm{H}]$ is linked to

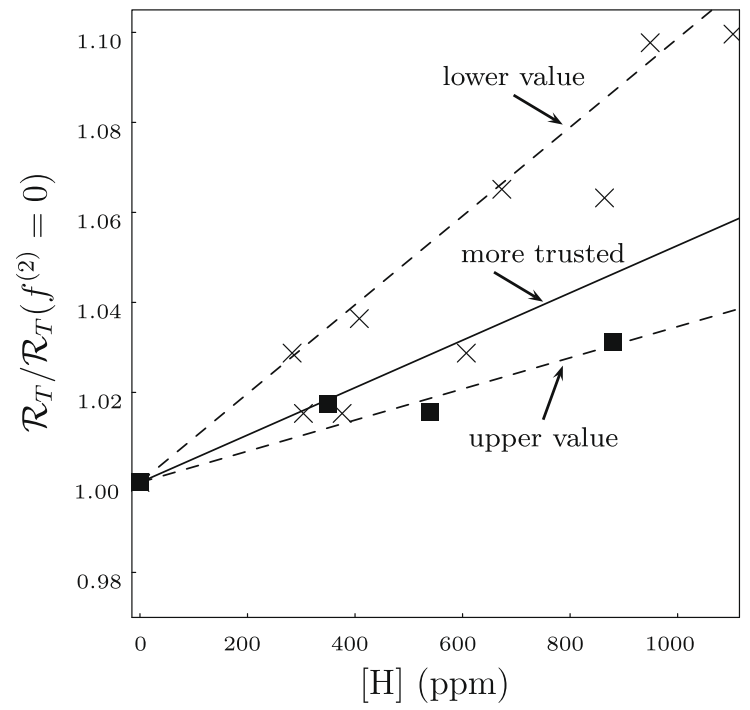

Fig. 12. Identification of the transverse aspect ratio $w_{T}$ : evolution of the normalized longitudinal ultimate tensile stress with respect to the hydrogen content (in ppm). Comparison between experimental data (square: Kuroda et al. (2002); cross: Grange et al. (2000)) and predictions of the proposed model ('lower value': $w_{T}^{\text {low }}=0.1$; 'more trusted value': $w_{T}^{\text {trust }}=0.25$; 'upper value': $w_{T}^{u p}=1.0 ; w_{L}=w_{T}$ in all cases, $\alpha=1$ ). 
the volume fraction of hydrides by: $[H] \simeq(1.66 \times$ $\left.10^{6} / 91\right) f^{(2)}$. The following transverse aspect ratios are exhibited:

(1) an upper value: $w_{T}^{u p}=1.0$,

(2) a lower value: $w_{T}^{\text {low }}=0.1$,

(3) a more trusted value: $w_{T}^{\text {trust }}=0.25$,

which correspond respectively to a lower value, an upper value and a 'best-estimate' value of the normalized transverse ultimate tensile stress. This bounding-estimating approach has been conducted in order to overcome some uncertainties on the experimental data: no clear informations are given about the aspect ratio of the hydrides in the sheets of Zircaloy tested by Kuroda et al. (2002), and the material tested by Grange et al. (2000) is slightly different (recrystallized state). Moreover, due to these uncertainties, no particular value was retained for the longitudinal aspect ratio $w_{L}$, and the simple choice $w_{L}=w_{T}$ was made for this identification of $w_{T}$. We checked that this choice has no significant influence on the transverse tensile stress (orthoradial loading) investigated at this stage.

\subsection{Longitudinal-to-transverse behavior, evolution of the plastic anisotropy of the matrix}

Since the transverse aspect ratio $w_{T}$ has been boundedestimated on transverse loadings, the longitudinal aspect ratio $w_{L}$ is now identified comparing the longitudinal response of hydrided Zircaloy to the transversal one. The experimental database PROMETRA (Balourdet et al., 1999; Yvon et al., 2001; Desquines et al., 2004; Cazalis et al., 2007) has shown that, for a cladding tube containing about $500 \mathrm{ppm}$ of hydrogen $\left(f^{(2)} \simeq 3 \%\right)$, the ultimate tensile stresses are nearly the same in the $\theta$ and $z$ directions, whereas the uniform elongation in the $z$ direction is about twice the one in the $\theta$ direction, namely:

$\mathscr{R}_{T} \simeq 0.97 \mathscr{R}_{L} \quad$ (ultimate tensile stress),

$\mathscr{A}_{T} \simeq(0.5-0.6) \mathscr{A}_{L} \quad$ (uniform elongation).

Another time, this experimentally observed overall transverse-to-longitudinal anisotropy is induced both by the plastic anisotropy of the matrix and by the shape and spatial distribution of the inclusions. Two remaining parameters affect this anisotropy: the longitudinal aspect ratio $w_{L}$, and the Hill-to-von Mises parameter $\alpha$. Focussing on the ultimate tensile stress, the influence of these two parameters is illustrated in Fig. 13 for a given value of the transverse inclusion aspect ratio $\left(w_{T}=w_{T}^{\text {trust }}\right)$ and $f^{(2)}=3 \%$. For a given degree of plastic anisotropy inside the matrix ( $\alpha$ fixed), the overall anisotropy decreases when the longitudinal-to-transverse inclusion aspect ratio $w_{L} / w_{T}$ increases. For a given inclusion shape $\left(w_{L} / w_{T}\right.$ fixed), the overall anisotropy increases with the parameter $\alpha$. This last remark is straightforward, recalling that when $\alpha=0$, the matrix follows a von Mises criterion. In conclusion, various couples $\left\{\alpha, w_{L} / w_{T}\right\}$ are appropriate candidates to mimic the experimental anisotropy (34).

Moreover, Fig. 13 indicates that, when $\alpha$ is greater than 0.1 , the experimental transverse-to-longitudinal ultimate

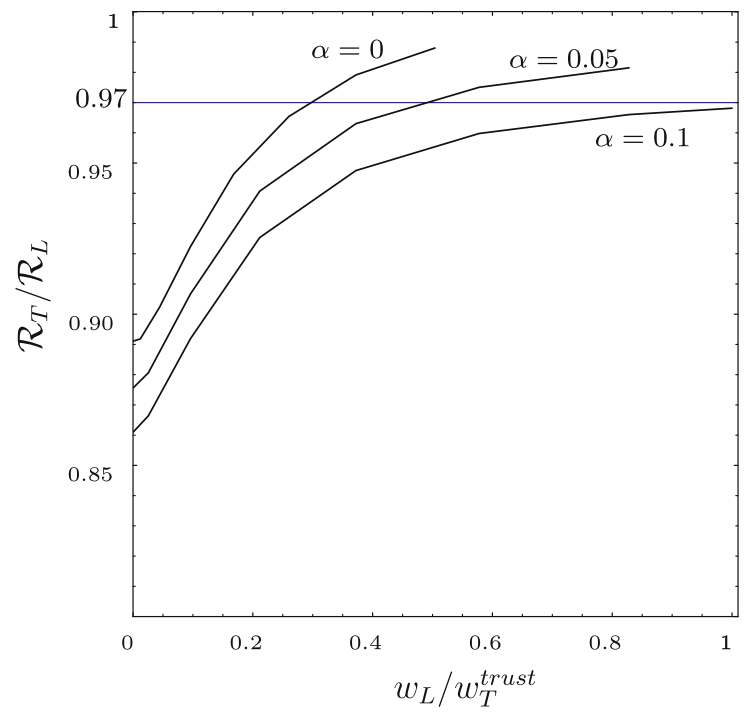

Fig. 13. Evolutions of transverse-to-longitudinal ultimate tensile stress $\mathscr{R}_{T} / \mathscr{R}_{L}$ with longitudinal-to-transverse inclusion aspect ratio $w_{L} / w_{T}$ for various values of the Hill-to-von Mises parameter $\alpha$ ( $w_{T}$ fixed to $\left.w_{T}^{\text {trust }}, f^{(2)}=3 \%\right)$.

tensile stress (34) cannot be obtained under the condition $w_{L} / w_{T} \leqslant 1$. Since hydrides are longer in the longitudinal direction than in the transversal direction (see Fig. 1), this condition has to be satisfied (most of experimental measurements suggest $w_{L} / w_{T} \simeq 0.1-0.2$ ). This last result, obtained for $w_{T}=w_{T}^{\text {trust }}$, shows qualitatively that the Hill-to-von Mises parameter $\alpha$ is lower than 0.1 for hydrided and irradiated Zircaloy cladding tubes containing about $500 \mathrm{ppm}$ of hydrogen. Fig. 14 confirms quantitatively this result for each estimatedbounded value of $w_{T}$. In other words, comparing the present modeling to the overall plastic anisotropy of highly irradiated and hydrided Zircaloy cladding tubes implies that the

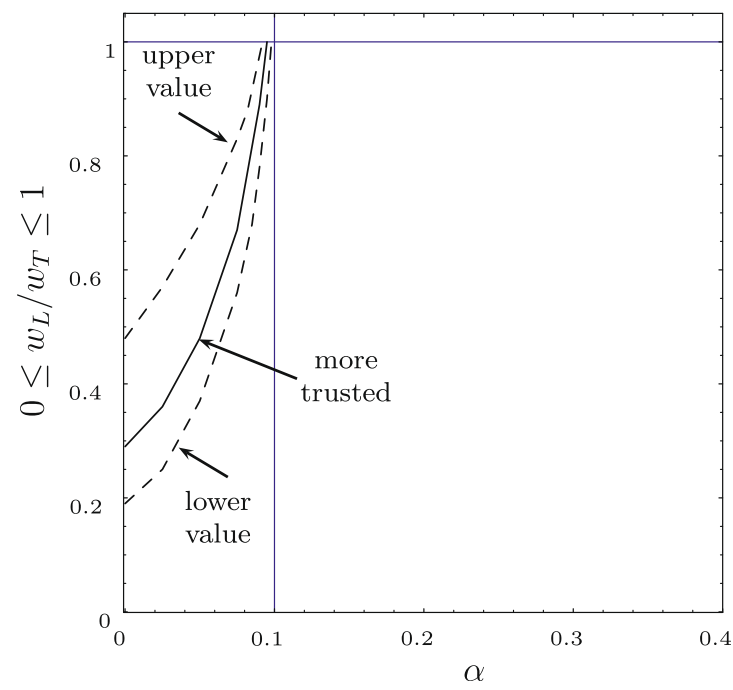

Fig. 14. Evolution of the longitudinal-to-transverse inclusion aspect ratio $w_{L} / w_{T}$ with respect to the Hill-to-von Mises parameter $\alpha$ corresponding to $\mathscr{R}_{T} / \mathscr{R}_{L}=0.97\left(f^{(2)}=3 \%\right)$. 
plastic anisotropy of the matrix phase decreases with the irradiation level. For irradiation corresponding to about 500 ppm mean hydrogen content (typically 5 cycle rods) inside a cladding tube, the matrix phase should have lost about $90 \%$ of its plastic anisotropy. This result is in agreement with some experimental measurements, which demonstrate qualitatively that the plastic anisotropy decreases with the neutron fluence (Nakatsuka and Nagai, 1987).

\subsection{Towards an evolution of the overall behavior with burn-up}

In order to predict the overall plastic behavior of the inpile Zircaloy, one has to incorporate in the present modeling a dependence of both the Hill-to-von Mises parameter $\alpha$ and the volume fraction $f^{(2)}$ on the burn-up. Sorting the effects of neutron irradiation and hydriding, Nakatsuka and Nagai (1987) show on Zircaloy-2 that the dependence of Knoop hardness increase on neutron fluence is similar to that of ultimate tensile stress. This result supplies experimental evolution of the ratio $\mathscr{R}_{T} / \mathscr{R}_{L}$ with the fluence $\phi$ (in $n / \mathrm{cm}^{2}$ ) without hydrides $\left(f^{(2)}=0\right.$ ). The case $\phi=0$ corresponds to $\alpha=1$ in the present model. Moreover we propose on the basis of the PROMETRA database to link the fluence to the burn-up $(B U)$ as:

$\phi\left(10^{21} \mathrm{n} / \mathrm{cm}^{2}\right)=0.18 B U(\mathrm{GWd} / \mathrm{tU})$.

The unit GWd/tU measures the fuel burn-up rate (Giga Watt days per tonne of initial uranium). As already mentioned, the irradiation dose is suspected to reduce the plastic anisotropy of the matrix. Since the degree of anisotropy of the matrix is linked in the model to the Hill-to-von Mises parameter $\alpha$ (the plastic anisotropy decreases with $\alpha$ ), we suggest here a decrease of $\alpha$ with the burn-up. The following exponential decrease seems adequate:

$\alpha=e^{-6 \times 10^{-2} B U}$.

Further investigations are required to definitively confirm this last relation. The resulting evolution of the transverse-to-longitudinal ultimate tensile stress ratio $\mathscr{R}_{T} / \mathscr{R}_{L}$ with the burn-up is given in Fig. 15, and compared to the experimental results of Nakatsuka and Nagai (1987).

Moreover, it is interesting for practical purpose to couple the effects of neutron irradiation and hydriding. In that case, both parameter $\alpha$ and hydride volume fraction $f^{(2)}$ depend on the burn-up. Based on the PROMETRA database, relation (36) is then completed by:

$f^{(2)}=5 \times 10^{-4} B U(\mathrm{GWd} / \mathrm{tU})$.

Again, we underline that the so-called 'effects of irradiation' - as employed in the nuclear industry context - combine both neutron irradiation effects and hydriding ones. The proposed approach is able to model these effects separately or simultaneously.

\section{Conclusion}

An estimate of the effective behavior of hydrided and irradiated Zircaloy-4 cladding tubes has been put forward. This effective behavior is elastoplastic and anisotropic. It takes into account an elastoplastic and orthotropic matrix

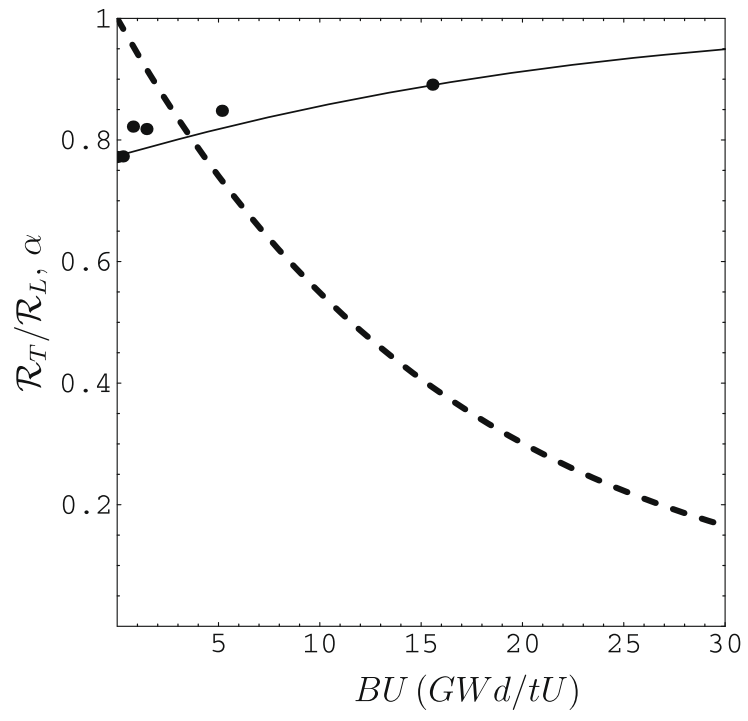

Fig. 15. Evolution of $\mathscr{R}_{T} / \mathscr{R}_{L}$ with the burn-up (line) for an exponential decreases of $\alpha$ with the burn-up (dashed line). Ratio of the Knoop hardness in two different sections and directions due to the experimental data of Nakatsuka and Nagai (1987) (points): results of tests on a normal to $e_{z}$ section with the indenter diagonal in the radial direction to results of tests on normal to $e_{r}$ section with the indenter diagonal in the longitudinal direction $\left(f^{(2)}=0\right)$.

with a power law and linear elastic and isotropic inclusions in the form of aligned platelets whose centers are isotropically distributed. The modified secant method of Ponte Castañeda and Suquet (1998) has been implemented using the estimate of Ponte Castañeda and Willis (1995) for the effective behavior of the linear comparison composite. The final model is based on morphological parameters which have been identified with the help of three-dimensional periodic numerical simulations.

The application of this model to highly irradiated Zircaloy-4 cladding tubes has proved that the experimentally measured overall plastic anisotropy of this material can only be obtained with a matrix having a degree of isotropy higher than the one of the 'fresh' Zircaloy matrix. When applied to highly irradiated cladding tubes, assuming that irradiation does not affect the initial mechanical properties of the phases, the model exhibits a much more anisotropic response than experimental results. However, comparisons between model and experiments give better results when the plastic anisotropy of the Zircaloy matrix is assumed to decrease with irradiation. For 5 cycle rods, we found that the Zircaloy-4 matrix phase should have lost about $90 \%$ of its initial plastic anisotropy.

\section{References}

Aitchison, I., 1969. Applications-related phenomena in zirconium and its alloys. In: ASTM-STP 458. ASTM, Philadelphia, PA, p. 160.

Arsène, S., 1997. Effet de la microstructure et de la température sur la transition ductile-fragile des Zircaloy hydrurés. Ph.D. Thesis, Ecole Centrale Paris.

Balourdet, M., Bernaudat, C., Basini, V., Hourdequin, N., 1999. The PROMETRA Program: assessment of mechanical properties of Zircaloy 4 fuel properties during an RIA. In: Transactions of the 
15th International Conference on Structural Mechanics in Reactor Technology (SMiRT-15), vol. II, Seoul, Korea, August, pp. 485-492.

Bornert, M., Suquet, P., 2001. Propriétés non linéaires des composites : approche par potentiels. In: Bornert, M., Bretheau, T., Gilormini, P. (Eds.), Homogénéisation en mécanique des matériaux, vol. 2. Hermès Science, pp. 45-88 (chapter 2).

Cazalis, B., Desquines, J., Poussard, C., Petit, M., Monerie, Y., Bernaudat, C. Yvon, P., Averty, X., 2007. The PROMETRA program: fuel cladding mechanical behavior under high strain rate. Nuclear Technology 157 215-229.

Coleman, C., Ambler, J., 1977. Susceptibility of zirconium alloys to delayed hydrogen cracking. In: Lowe, A., Jr., Parry, G. (Eds.), Zirconium in the Nuclear Industry. ASTM STP 633, American Society for Testing and Materials, pp. 589-607.

Coleman, C., Hardie, D., 1966. The hydrogen embrittlement of alphazirconium - a review. Journal of Less-Common Metals 11, 168-185.

Delobelle, P., Robinet, P., Geyer, P., Bouffioux, P., 1996. A model to describe the anisotropic viscoplastic behavior of Zircaloy-4 tubes. Journal of Nuclear Materials 238, 135-162.

Desquines, J., Cazalis, B., Bernaudat, C., Poussard, C., Averty, X., Yvon, P., 2004. Zircaloy-4 fuel cladding mechanical behavior in the field of RIA transients through the PROMETRA program. In: Proceedings of the ASTM conference on Zirconium in the Nuclear Industry. Stockholm, Sweden, June.

Desquines, J., Fédérici, E., 2001. Propriétés mécaniques en traction monotone du Zircaloy-4 irradié à haute vitesse de déformation. Tech. Rep., IRSN, SEMAR/LEC 01/99.

Dutton, R., Nuttall, K., Puls, M., Simpson, L., 1977. Mechanisms of hydrogen induced delayed cracking in hydride forming materials. Metallurgical Transactions A 8A, 1553-1562.

Ells, C., 1968. Hydride precipitates in zirconium alloys (a review). Journal of Nuclear Materials 28, 129-151.

Fandeur, O., 2001. Etude expérimentale et modélisation mécanique de la corrosion sous contrainte des gaines en Zircaloy-4. Ph.D. Thesis, Ecole Centrale Paris.

Fandeur, O., Pilvin, P., Prioul, C., 2001. Modélisation du comportement mécanique du Zircaloy-4. Journal of Physics IV 11, 109-118.

Froes, F., Yau, T.-L., Weidinger, H., 1996. Titanium, zirconium and hafnium. In: Cahn, R., Haasen, P., Kramer, E. (Eds.), Structure and properties of nonferrous alloys, Materials Science and Technology: A Comprehensive Treatment, vol. 8. Wiley-VCH, pp. 398-468.

Garde, A., 1989. Effects of irradiation and hydriding on the mechanical properties of zircaloy-4 at high fluence. In: Van Swam, L., Eucken, C. (Eds.), Zirconium in Nuclear Industry: Eighth International Symposium (ASTM STP 1023). American Society for Testing and Materials, Philadelphia, pp. 548-569.

Gómez, M., Domizzi, G., López Pumarega, M., Ruzzante, J., 2006. Characterization of hydrogen concentration in Zircaloy-4. Journal of Nuclear Materials 353, 167-176.

Grange, M., Besson, J., Andrieu, E., 2000. An anisotropic gurson type model to represent the ductile rupture of hydrided Zircaloy-4 sheets. International Journal of Fracture 105, 273-293.

Huang, F., Mills, W., 1991. Delayed hydride cracking behavior for zircaloy2 tubing. Metallurgical Transactions A 22A, 2049-2060.

Kammenzind, B., Franklin, D., Peters, H., Duffin, W., 1996. Hydrogen pickup and redistribution in alpha-annealed zircaloy-4. In: Bradley, E., Sabol, G. (Eds.), Zirconium in Nuclear Industry: Eleventh International Symposium (ASTM STP 1295). American Society for Testing and Materials, Garmisch-Partenkirchen, Germany, pp. 338-369.

Kearns, J., 1967. Terminal solubility and partitioning of hydrogen in the alpha phase of zirconium, zircaloy-2 and zircaloy-4. Journal of Nuclear Materials 22, 292-303.

Kuroda, M., Yamanaka, S., Setoyama, D., Uno, M., Takeda, K., Anada, H., Nagase, F., Uetsuka, H., 2002. Tensile test of hydrided Zircaloy. Journal of Alloys and Compounds, 404-407.

Leclercq, S., Rousselier, G., Cailletaux, G., 2007. A generic method for modeling the behavior of anisotropic metallic materials: application to recrystallized zirconium alloys. Mechanics of Materials 39, 458-472.

Lee, K., Hong, S., 2002. Zirconium hydrides and their effect on the circumferential mechanical properties of $\mathrm{Zr}-\mathrm{Sn}-\mathrm{Fe}-\mathrm{Nb}$ tubes. Journal of Alloys and Compounds 346, 302-307.
Li, G., Ponte Castañeda, P., 1994. Variational estimates for the elastoplastic response of particle-reinforced metal-matrix composites. Applied Mechanics Reviews 47, S77-94.

Lufrano, J., Sofronis, P., 2000. Micromechanics of hydride formation and cracking in zirconium alloys. Computer Modeling in Engineering and Sciences 1, 119-131.

Masson, R., 2008. New explicit expressions of the Hill polarization tensor for general anisotropic elastic solids. International Journal of Solids and Structures 45, 757-769.

Marino, G., 1972. A numerical calculation of the redistribution of an interstitial solute in a thermal gradient. Nuclear Science and Engineering 49, 93-98.

McMinn, A., Darby, E., Schofield, J., 2000. In: Sabol, G., Moan, G. (Eds.), Zirconium in Nuclear Industry: Twelfth International Symposium (ASTM STP 1354). American Society for Testing and Materials, Toronto, Canada, pp. 173-195.

Michel, J.-C., Moulinec, H., Suquet, P., 2001. Composites à microstructure périodique. In: Bornert, M., Bretheau, T., Gilormini, P. (Eds.), Homogénéisation en mécanique des matériaux, vol. 1. Hermès Science, pp. 57-94.

Mücke, R., Bernhardi, O.-E., 2003. A constitutive model for anisotropic materials based on the Neuber's rule. Computer Methods in Applied Mechanics and Engineering 192, 4237-4355.

Mura, T., 1982. Micromechanics of Defects in Solids, 1987th ed. Martinus Nijhoff, Dordrecht.

Nakatsuka, M., Nagai, M., 1987. Reduction of plastic anisotropy of Zircaloy cladding by neutron irradiation. Journal of Nuclear Science and Technology 24 (10), 832-838.

Northwood, D., Kosasih, U., 1983. Hydrides and delayed hydrogen cracking in Zirconium and its alloys. International Metals Review 28, 92-121.

Perovic, V., Weatherly, G., 1984. The nucleation of hydrides in a zr-2.5 wt\% nb alloy. Journal of Nuclear Materials 126, 160-169.

Ponte Castañeda, P., Suquet, P., 1998. Nonlinear composites. Advances in Applied Mechanics 34, 171-302.

Ponte Castañeda, P., Willis, J.R., 1995. The effect of spatial distribution on the effective behavior of composite materials and cracked media. Journal of the Mechanics and Physics of Solids 43, 1919-1951.

Ponte Castañeda, P., 1991. The effective properties of nonlinear isotropic composites. Journal of Mechanics and Physics of Solids 39, 45-71.

Sawatzky, A., 1960. Hydrogen in zircaloy-2: its distribution and heat of transport. Journal of Nuclear Materials 2, 321-328.

Shi, S., Puls, M., 1994. Criteria for fracture initiation at hydrides in zirconium alloys I. Sharp crack tip. Journal of Nuclear Materials 208, 232-242.

Suquet, P., 1995. Overall properties of nonlinear composites: a modified secant moduli theory and its link with Ponte Castañeda's nonlinear variational procedure. C.R. Acad. Sci., Paris série II (320), 563-571.

Suvorov, A., Dvorak, G., 2002. Rate form of the Eshelby and Hill tensors. International Journal of Solids and Structures 39, 5659-5678.

Une, K., Ishimoto, S., 2003. Dissolution and precipitation behavior of hydrides in zircaloy-2 and high fe zircaloy. Journal of Nuclear Materials 322, 66-72.

Varias, A., Massih, A., 2002. Hydride-induced embrittlement and fracture in metals - effect of stress and temperature distribution. Journal of Mechanics and Physics of Solids 50, 1469-1510.

Vizcaíno, P., Banchik, A., Abriata, J., 2002. Solubility of hydrogen in Zircaloy-4: irradiation induced increase and thermal recovery. Journal of Nuclear Materials 304, 96-106.

Wäppling, D., Massih, A., Stahle, P., 1997. A model for hydride-induced embrittlement in zirconium-based alloys. Journal of Nuclear Materials 249, 231-238.

Yamanaka, S., Yamada, K., Kurosaki, K., Uno, M., Takeda, K., Anada, H., Matsuda, T., Kobayashi, S., 2001. Thermal properties of zirconium hydride. Journal of Nuclear Materials 294, 94-98.

Yamanaka, S., Yoshioka, K., Uno, M., Katsura, M., Anada, H., Matsuda, T., Kobayashi, S., 1999. Thermal and mechanical properties of zirconium hydride. Journal of Alloys Compounds 293, 23-29.

Yvon, P., Sainte Catherine, C., Duguay, C., Carassou, S., Hourdequin, N. Cazalis, B., Bernaudat, C., 2001. Development of new techniques to assess the mechanical behaviour of Zircaloy-4 during an RIA. In: IAEA Technical Committee on Fuel Behavior Under Transient LOCA Conditions, Halden, Norway, September. 